\title{
Integrated PET/CT in the staging of nonsmall cell lung cancer: technical aspects and clinical integration
}

\author{
W. De Wever*, S. Stroobants”, J. Coolen* and J.A. Verschakelen*
}

ABSTRACT: Lung cancer is a common disease and is a leading cause of death in many countries. The management of lung cancer is directed by an optimal staging of the tumour. Integrated positron emission tomography (PET)/computed tomography (CT) is an anatomo-metabolic imaging modality that has recently been introduced to clinical practice and combines two different techniques: CT, which provides very detailed anatomic information; and PET, which provides metabolic information. One of the advantages of PET/CT is the improved image interpretation. This improvement can result in the detection of lesions initially not seen on CT or PET, a more precise location of lesions, a better characterisation of the lesion as benign or malignant and a better differentiation between tumour and surrounding structures. Initial studies demonstrate better results for PET/CT in the staging of lung cancer in comparison with PET alone, CT alone or visual correlation of PET and CT. The purpose of the present article is to discuss technical aspects of integrated PET/CT and to attempt to outline how to introduce integrated PET/ CT in clinical and daily practice.

KEYWORDS: Computed tomography, imaging, integrated positron emission tomography/ computed tomography, lung cancer, positron emission tomography, staging

ung cancer is a common disease with $\sim 1.3$ million new cases per year worldwide and is a leading cause of death in many countries. Nonsmall cell lung cancer (NSCLC) accounts for $75-80 \%$ of these cases [1]. Optimal staging is important in order to determine the best possible therapeutic option, to clarify operability and to have an idea about the outcome of the patient. Staging of NSCLC is based on tumour size and location (T-stage), nodal involvement (N-stage) and the presence or absence of metastases (M-stage) [2]. Computed tomography (CT) is the standard imaging technique for the investigation of lung cancer in most centres. Multidetector CT (MDCT) is the current technological standard technique that can provide improved information about transfissural tumour growth, pleural involvement and mediastinal and chest wall invasion. Over the past decade, ${ }^{18}$ fluorodeoxyglucose $\left({ }^{18} \mathrm{~F}\right.$-FDG)-positron emission tomography (PET) has proved to be a valuable noninvasive imaging technique for the assessment of nodal and extrathoracic disease. Its main limitation is the lack of spatial resolution. The first integrated PET/CT machine came into clinical practice in 1998, and it is inevitable that all future installations of PET machines will be in the form of PET/CT [3]. The additional information gained with integrated PET/CT can be: 1) detection of lesions initially not seen on $\mathrm{CT}$ or PET (fig. 1); 2) more precise location of lesions and a better delineation of the surrounding structures (fig. 2); and 3) better characterisation of the lesion as benign or malignant (fig. 3) [4]. Owing to the concept of integrated PET/CT, the technique of the CT component is somewhat different of those of a dedicated CT for chest and abdomen scanning. Recently, it has been shown that, in tumour staging of patients with lung cancer, analysis of integrated PET/CT images is superior to that of CT images alone, PET images alone, and PET and CT images viewed side by side $[5,6]$. The article will draw on the current authors' own experience and published studies in order to: detail the most important technical parameters concerning the CT component of
AFFILIATIONS

*Depts of Radiology, and

${ }^{\#}$ Nuclear Medicine, University Hospitals Gasthuisberg, Leuven, Belgium.

CORRESPONDENCE

W. De Wever

Dept of Radiology

University Hospitals Gasthuisberg Herestraat 49

3000 Leuven

Belgium

Fax: 3116434765

E-mail: walter.dewever@uzleuven.be

Received

March 062008

Accepted after revision:

July 012008

STATEMENT OF INTEREST

None declared. 

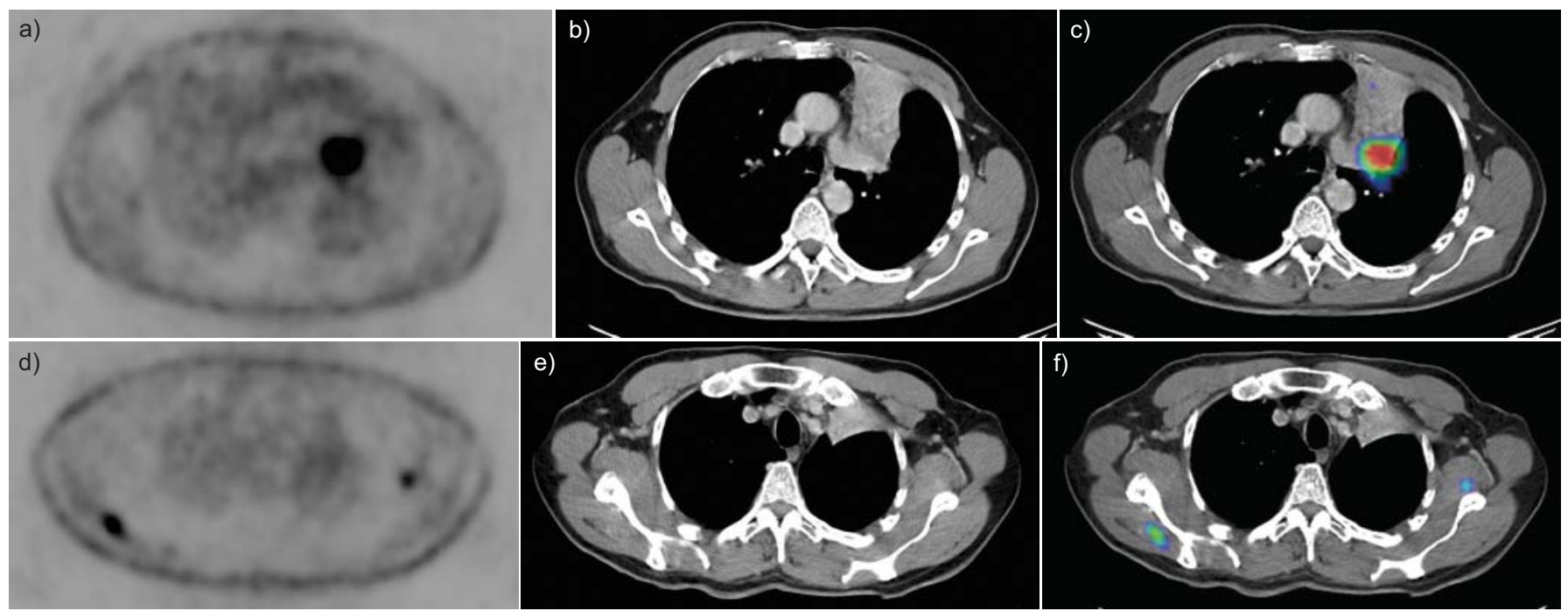

FIGURE 1. A 57-yr-old male with a cerebral metastasis from an unknown tumour. Integrated positron emission tomography (PET)/computed tomography (CT) showed a lung tumour in the left upper lobe with retro-obstructive atelectasis; PET/CT (c) delineated the tumour from the retro-obstructive atelectasis better than PET and CT alone ( $a$ and $b$ ). With CT no other lesions suspect for metastases or adenopathies can be demonstrated ( $b$ and e). PET showed some other ${ }^{18}$ fluorodeoxyglucose hotspots suggestive for bone metastasis and pathological lymph nodes in the left axilla (d). PET/CT ( $f$ ) showed that these hotspots were muscle metastases that were not retained initially on the CT images (e), but could be demonstrated retrospectively on these CT images.

integrated PET/CT; summarise the most important studies concerning staging of NSCLC with integrated PET/CT; and discuss how integrated PET/CT can be utilised in clinical practice.

\section{TECHNICAL ASPECTS OF INTEGRATED PET/CT \\ CT-based attenuation correction and related artefacts}

A typical whole-body PET scan is started $60 \mathrm{~min}$ after the intravenous administration of $\pm 4.5 \mathrm{MBq}{ }^{18} \mathrm{~F}$-FDG per $\mathrm{kg}$ body weight. The axial field of view of the PET system $(10-15 \mathrm{~cm})$ is extended by imaging in multiple bed positions to cover the whole body [7]. An acquisition time of 4-6 min per bed position produces images of relatively good resolution and contrast, in a total imaging time of $30-40 \mathrm{~min}$. When quantitative assessment of FDG metabolism is needed, e.g. for the assessment of the metabolic response to an antineoplastic treatment, correction for soft tissue attenuation is crucial. To achieve this attenuation correction, a set of corresponding images (transmission scan) is acquired with an external high-energy photon source (germanium-68 or caesium-137). This transmission scan adds $\geqslant 50 \%$ to the scanning time and results in data with a relatively high noise level [8]. This transmission scan can be performed prior to injection of the tracer (cold transmission) or afterwards (hot transmission).

One of the advantages of an integrated PET/CT system is that the CT data can be used for attenuation correction because the CT scan can be used as a transmission scan. To use the CT scan for attenuation correction, the attenuation values of low-dose CT energies must be scaled to high-dose (511 keV) PET energies using scaling algorithms. CT-based attenuation correction in integrated $\mathrm{PET} / \mathrm{CT}$ results in a lower noise emission scan and faster examinations (reduction of wholebody scan times by at least $40 \%$ ) and, thus, fewer motion artefacts and a higher throughput [9]. A number of practical aspects using CT-based attenuation correction, such as patient respiration, the use of i.v. and p.o. CT contrast media, and the presence of catheters and other metal objects in the patient, could potentially generate artefacts, because the scaling algorithms that must scale the low-dose energies of CT into the high-dose PET energies are developed for normal soft tissue densities.
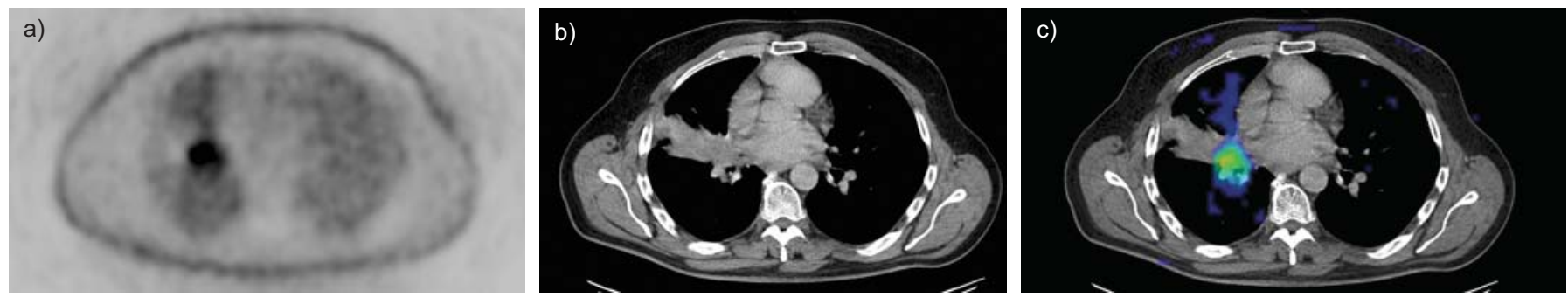

FIGURE 2. A 54-yr-old male with a central lung tumour in the right lung. a) Positron emission tomography (PET) showed ${ }^{18}$ fluorodeoxyglucose uptake in the lung tumour but not or discrete in the retro-obstructive atelectasis. b) Computed tomography (CT) showed a central lung tumour with retro-obstructive atelectasis. The central tumour cannot be delineated from the retro-obstructive atelectasis on this CT without intravenous contrast administration. C) PET/CT images can more easily delineate the central lung tumour from the retro-obstructive atelectasis. 

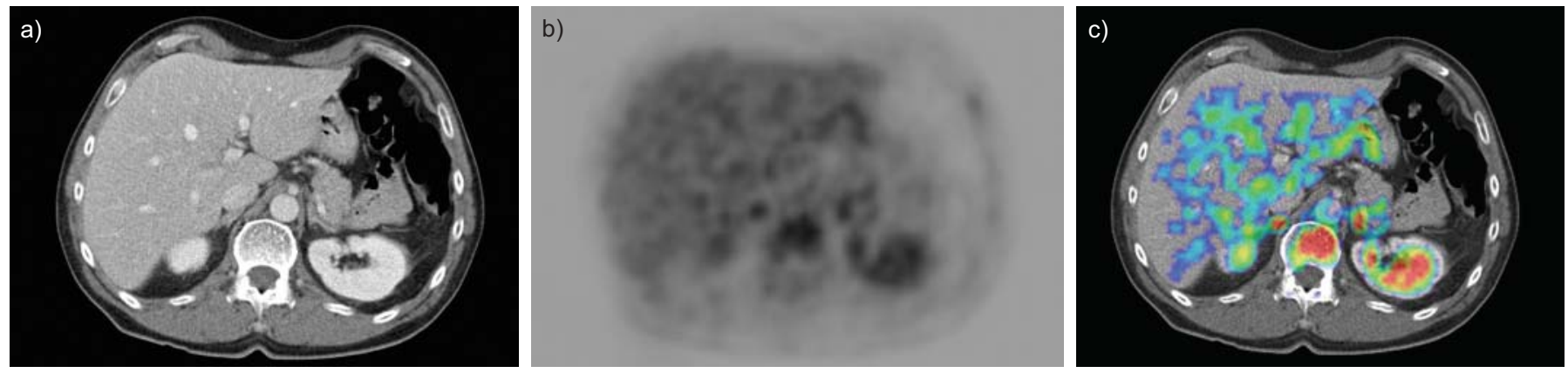

FIGURE 3. A 54-yr-old man with a lung tumour. Computed tomography (CT) showed no suspicious lesions in the adrenal glands (a), however PET showed a hotspot in the region of the left adrenal gland (b). c) Only with integrated PET/CT can the hotspot be exactly located on the adrenal gland, resulting in the conclusion that this adrenal gland is suspect for metastatic disease.

\section{Respiration artefacts}

Attenuation correction of PET images with helical CT in integrated PET/CT matches only the spatial resolution of CT and PET and not the temporal resolution. A respirationaveraged $\mathrm{CT}$ is thought to match the temporal resolution of CT and PET. The acquisition time for a conventional PET emission scan amounts to several minutes per field of view and represents an average over many breathing cycles. As the PET data are acquired during tidal breathing, an average diaphragm position is represented. CT-based attenuation correction can be thought of as a snapshot of the patient's body during a certain respiration point. Due to the rapid acquisition of the $\mathrm{CT}$, the diaphragm is mapped in a certain position that may be different from that obtained with PET.

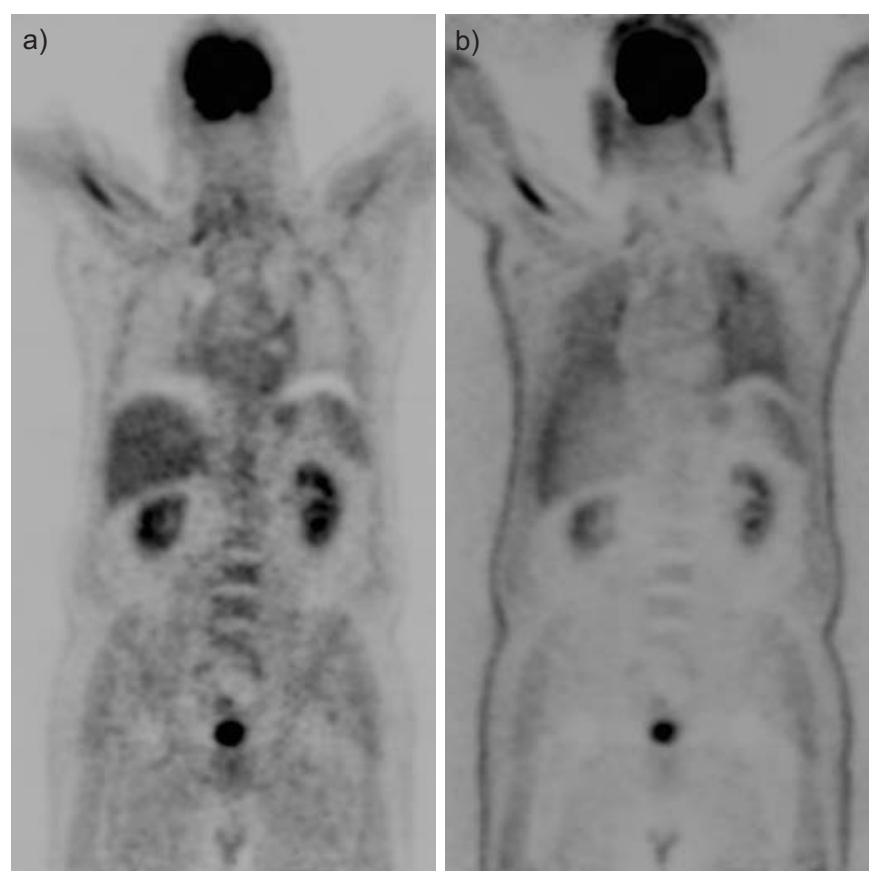

FIGURE 4. Cold artefacts on computed tomography (CT)-based attenuationcorrected positron emission tomography (PET) images. CT-based attenuationcorrected images (a) showed white curvilinear areas above the liver and spleen (cold artefacts): regions without information due to respiration artefacts. The attenuation uncorrected PET images (b) do not show these artefacts and can be used to exclude false-positives or false-negatives.
The acquisition of data during a very brief time can lead to differences between the CT attenuation data and the PET emission images [10], and these inevitable differences in respiratory cycles between the PET and CT images may potentially result in mislocation of lesions. The discrepancy in the diaphragmatic position between PET and CT can result in the appearance of a "cold" artefact at the lung base (fig. 4) [11] Because the caudal and peripheral parts of the lung have a larger range of respiration-induced movement than that of the central regions of the lung or the apices, lesions in the periphery and in the base of the lung exhibit larger mismatches of integrated PET/CT co-registration than those of lesions that are located in the apices or in the central regions of the lung [12]. GoERrEs et al. [13] have found that optimal image fusion occurred when CT data sets were acquired during normal expiration. Similar studies also have shown a reduction in the severity and frequency of respiratory motion artefacts when a limited expiratory breath-hold protocol was used [14, 15]. GILMAN et al. [10] have found that for integrated PET/CT of the chest, excellent image fusion was achieved during expiration, mid-suspended breath-hold and quiet breathing, with no significant variation in scoring of anatomic alignment. Following the results of these studies, a normal expiration breath-hold protocol for CT scanning is recommended in order to obtain an attenuation map that matches the PET emission image.

\section{High-density attenuation artefacts}

The presence of foreign materials with higher density levels than normal tissues may result in an overestimation of the attenuation values and also, in return, overestimation of the FDG uptake. These focal artefacts may mimic true lesions (false-positives) or may lead to the false-negative interpretation of a lesion as an artefact close to metallic objects [12]. A review of the attenuation uncorrected PET images allows for the differentiation between true tracer uptake and artefact. The absence of increased activity on the uncorrected images in contrast to the corrected images confirms an area of apparently increased tracer uptake rather than a "true" lesion (fig. 5) [16].

The use of i.v. and p.o. CT contrast agents in CT protocols has demonstrated substantial benefits: improved delineation of anatomic structures; increased sensitivity for detection of pathological lesions; and improved accuracy in lesion characterisation [17, 18]. Therefore, most CT protocols in daily 

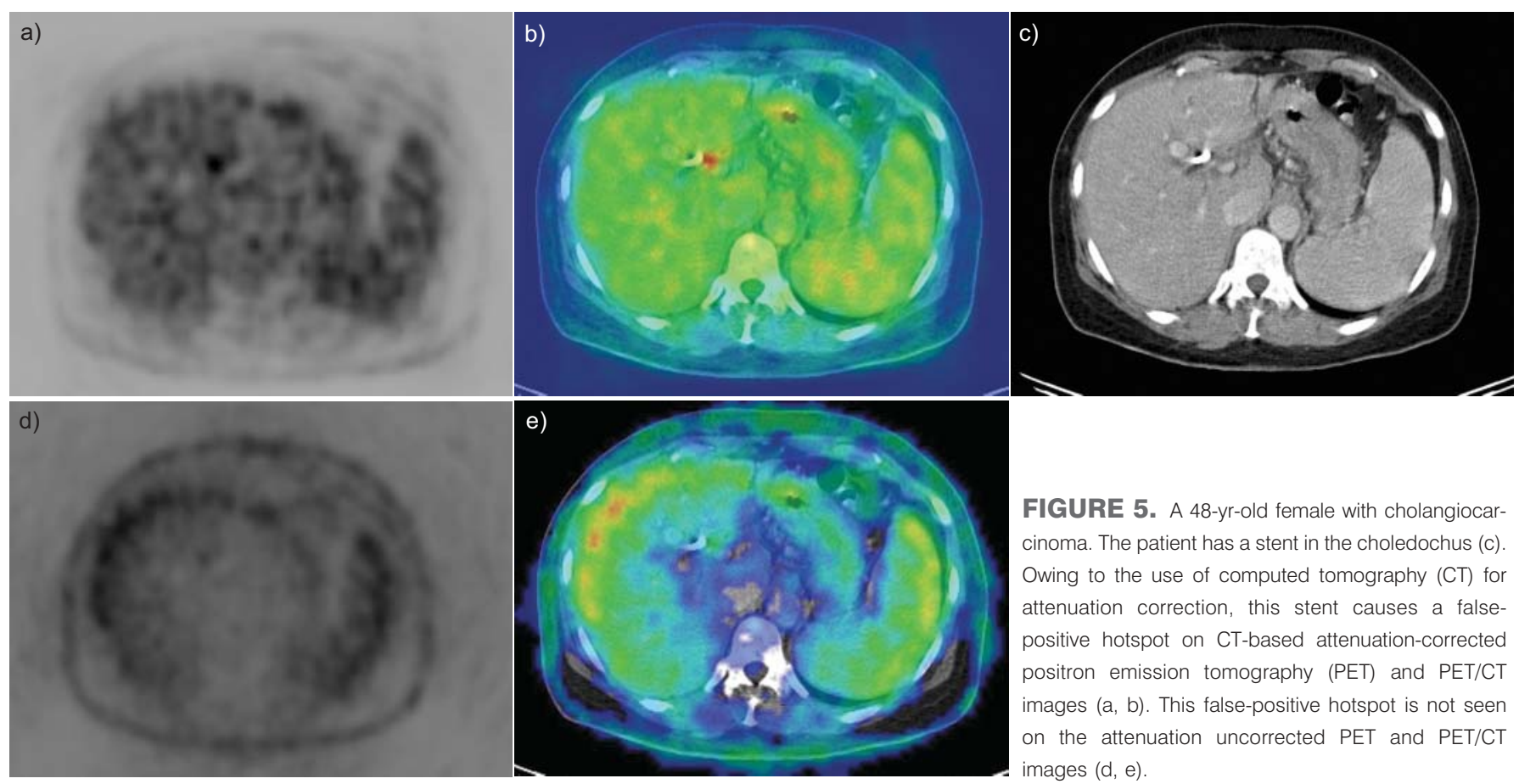

FIGURE 5. A 48-yr-old female with cholangiocarcinoma. The patient has a stent in the choledochus (c). Owing to the use of computed tomography (CT) for attenuation correction, this stent causes a falsepositive hotspot on CT-based attenuation-corrected positron emission tomography (PET) and PET/CT images $(a, b)$. This false-positive hotspot is not seen on the attenuation uncorrected PET and PET/CT images $(d, e)$.

radiological routine include contrast-enhanced images based on the application of oral and i.v. contrast agents. The use of i.v. and p.o. contrast when performing integrated PET/CT is a subject of dispute, since it raises questions about the production of artefacts on the PET images [9]. This concern is theoretically justified, since the algorithms that convert the CT attenuation coefficients measured at a range of $40-140 \mathrm{keV}$ to the $511 \mathrm{keV}$ values for emission data correction were developed and validated mainly for unenhanced human tissue densities [8]. Previous studies have shown that scaling errors, although small (mean maximum standardised surface uptake value difference 5-7\%), can occur in areas with high iodine concentration (e.g. liver, spleen and aorta) on CT acquisition [19]. As a result of the CT-based attenuation correction when scaling algorithms are used, the resulting overestimation may occur and may lead to image artefacts in the emission data. These artefacts appear as areas of apparently increased glucose metabolism on CT-based attenuation-corrected PET images in co-registration with areas of high contrast concentration [19].

Local accumulation of oral contrast agents that is caused either by ingestion, by a delay in the intestinal passage or by a different distribution of the contrast material owing to the time interval ( $\sim 10 \mathrm{~min}$ ) between the acquisition of CT and PET, can introduce PET artefacts if CT-based PET attenuation correction is performed [20]. However, when the contrast agent is distributed homogeneously in the bowel, the effect of oral CT contrast agents on the standardised surface uptake value seems to be negligible. DiZENDORF et al. [21] have reported only a $4 \%$ overestimation of the related standardised surface uptake value when evaluated in clinical routine. When there is an interpretation problem due to the use of p.o. (and also i.v.) contrast agents, attenuation uncorrected images can be used to solve these problems, since artefacts will be found only on attenuation-corrected data.

\section{CT image quality in integrated PET/CT}

\section{CT dose}

An important consideration is which CT dose to choose for the integrated PET/CT protocol. For a diagnostic CT, about $140 \mathrm{kV}$ and $120 \mathrm{~mA}$ are normally used to obtain images of optimum quality. Due to the concept of integrated PET/CT where a whole body CT scan from the top of the skull till the upper part of the tights is made, this dose cannot be generated with the first integrated PET/CT scanners. HANY et al. [22] have compared different CT doses (140 kV for all scans but varying current: 10, 40, 80 and $120 \mathrm{~mA}$ ). In the study conducted by HANY et al. [22], 21\% of all lesions were classified as undecided with PET alone and, thus, could not be specified. By using lowdose CT (10-40 mA) for image co-registration, an additional $7 \%$ of all lesions could be classified correctly and this was a result of these lesions being located more accurately. This reduction in false-negative results significantly increased the accuracy of integrated PET/CT compared with that of PET alone. When an 80-mA CT was used, the number of undecided lesions was reduced to $12 \%$. However, using a $120-\mathrm{mA} \mathrm{CT}$ did not further improve lesion classification. Therefore, the authors concluded that PET/80-mA CT should be used for optimal reduction of the number of undecided lesions. In addition, PET / 80-mA CT made a correct diagnosis of disease in $90 \%$ of the cases. Thus, it is feasible that an $80-\mathrm{mA}$ scan is incorporated as a routine protocol in diagnostic integrated PET/CT.

Integrated PET/CT examinations incur an increased patient exposure compared with an individual staging CT or PET examination. The patient effective dose depends on the scan parameters (tube current and potential, pitch factor and slice 
collimation), on scanner-specific factors (beam filtration and beam-shaping filter) and on the length of the scan region [23]. Reduction in tube current is the most practical means of reducing CT radiation dose. A 50\% reduction in tube current reduces radiation dose by half. Any decrease in tube current should be considered carefully, because such reduction causes an increase in image noise, which may affect the diagnostic outcome of the examination [24]. Therefore, a high-dose CT scan $(85 \mathrm{~mA})$ can be used for full diagnostic evaluation. However, a low-dose CT scan $(40 \mathrm{~mA})$ can only be used for attenuation correction and for correlation.

Tube potential determines the X-ray beam energy, and variation in tube potential causes a substantial change in CT radiation dose. Tube voltage affects both image noise and tissue contrast. For very large patients, a higher tube voltage is generally more appropriate. Further research on the use of lower tube voltage for dose advantages is warranted.

For spiral CT scanners, pitch is defined as the ratio of table feed per gantry rotation to the nominal width of the X-ray beam. Faster table speed for a given collimation, resulting in a higher pitch, is associated with a reduced radiation dose [24]. In MDCT with more than two detector rows, each detector contributes to every reconstructed image [25]. Owing to "overbeaming" in MDCT, some amount of the X-ray beam is incident beyond the edge of the detector rows. Generally, thicker beam collimation in MDCT results in a more doseefficient examination. However, thick collimation limits the width of the thinnest sections that can be reconstructed.

With the widespread availability of spiral CT scanners, there is a general tendency to increase the area of coverage. In integrated PET/CT, a total body $\mathrm{CT}$ is introduced. A larger scan area increases effective radiation dose to the patient. Whenever clinically justifiable, the range of whole-body scan should be limited by the symphysis at the lower limit and should exclude the eye lenses from the cranial imaging range [24]. The effective patient dose determined for different PET/ CT protocols are summarised in table 1 .

\begin{tabular}{ll} 
TABLE 1 & $\begin{array}{l}\text { Patient effective dose for whole-body low- and } \\
\text { high-dose positron emission tomography (PET)/ } \\
\text { computed tomography (CT) protocol }\end{array}$ \\
Protocol & Effective dose mSv \\
\hline Low dose & 0.2 \\
Topogram & 1.3 \\
40-mA CT & 7.0 \\
PET, 370 MBq ${ }^{18}$ F-FDG & 8.5 \\
Global examination & 0.2 \\
High dose & 17.6 \\
Topogram & 7.0 \\
85-mA CT & 24.8 \\
PET, 370 MBq ${ }^{18}$ F-FDG & \\
Global examination & \\
\hline &
\end{tabular}

\section{What kind of CT is needed?}

Stage-adapted treatment in oncology relies on correct tumour staging for patients with NSCLC. MDCT has become widely used and can be considered to be the imaging of choice. From the radiologist's point of view, fully diagnostic CT requires the following: sufficient spatial resolution with an acceptable signal-to-noise ratio; administration of an appropriate amount of oral or i.v. contrast material if there are no contraindications; and a radiation dose as low as reasonably achievable to obtain diagnostic information [26].

The question of interest to the radiologist is: what is the degree of image quality achieved in integrated PET/CT imaging? Today, different approaches are adopted for integrated PET/ CT scanning, with the CT protocol positioned between two possible extremes: 1 ) in which CT is used as a fast transmission source for attenuation correction and approximate anatomical mapping, and is performed with a low radiation dose (lowdose $10-40 \mathrm{~mA} \mathrm{CT}$ ); or 2) in which CT is used for both attenuation correction and diagnostic purposes, being performed with a standard radiation dose and i.v. and oral contrast (diagnostic $\geqslant 80 \mathrm{~mA} \mathrm{CT}$ ) [27]. With regard to the questions of whether we need oral and/or i.v. CT contrast agents, or if FDG is the new radiological contrast agent, the answers are not very clear at the present moment. ANTOCH et al. [18] have proposed different CT protocols in a study of CT with or without contrast agents, depending on what kind of information is needed and whether there is already a diagnostic CT available.

\section{Normal variants and pitfalls in FDG imaging}

Many benign lesions that have increased glucose metabolism can accumulate FDG and can be misinterpreted as malignant. In addition, physiological uptake of FDG, normal variants and findings related to patients' medical and surgical histories can also lead to a false-positive interpretation, which of course can influence conclusions on staging and management [28].

Physiological uptake of FDG is seen in the brain, heart, and gastrointestinal and genitourinary tracts. When physical activity in striated muscles is increased or when subconscious contraction due to, for example, anxiety, occurs shortly before or during the FDG uptake phase takes place, FDG accumulation can also be seen. The muscle groups commonly affected are located in the head and neck. The uptake pattern is typically bilateral, symmetric, fusiform or elongated, and is seldom confused with malignancy. Nevertheless, asymmetric muscle uptake can occur. Brown fat, another potential source of false-positive interpretation, plays an important role in thermogenesis associated with increased glucose uptake. Brown fat is abundant in children and is located in the cervical, axillary, paravertebral, mediastinal and abdominal regions [12]. Brown fat deposits diminish with age because of reduced demands for thermogenesis, making brown fat unusual in adults; however, it can been more often in winter time and in thin patients. Typically, the increased FDG uptake in brown fat is bilateral and symmetric and is seldom confused with malignancy, although FDG uptake can be asymmetric or focal in the mediastinum and abdomen, mimicking malignancy.

FDG uptake can occur in inflammatory processes and atherosclerotic disease. An infectious and inflammatory 
condition has been reported to be caused by increased glycolysis in leukocytes, lymphocytes and macrophages (fig. 6) [29]. Inflammation associated with atherosclerotic disease of the aorta and its major branches can show FDG uptake [30]. This FDG uptake is usually low grade and has a linear configuration. Occasionally, atherosclerotic plaques manifest as focal areas of increased FDG uptake that can be misinterpreted as malignancy.

Iatrogenic causes of focal or diffuse FDG uptake include: granulation tissue; healing wounds; talc deposits in the pleura after pleurodesis; placement of central lines, chest tubes and gastrotomy tubes; percutaneous needle biopsy; and mediastinoscopy; as well as FDG iatrogenic microembolism [28, 31]. In most cases, the use of CT to locate these foci of increased FDG uptake accurately is sufficient in preventing misinterpretation.

Benign tumours such as sclerosing haemangioma, leiomyoma and inflammatory pseudotumour may show increased FDG uptake. Some malignant lesions can lead to false-negative interpretations due to little FDG uptake on integrated PET/CT images. Mucinous and nonmucinous bronchoalveolar carcinoma (BAC), adenocarcinomas with BAC components, carcinoids and mucoepidermoid carcinomas are all tumours that may show little FDG uptake and, thus, may simulate benign tumours [32].

\section{DIAGNOSIS AND STAGING}

\section{Tumour assessment (T-stage)}

CT plays an important role in the T-staging of patients with NSCLC. Due to the increased image quality, the recently introduced MDCT scanners can depict with greater confidence an invasion of a tumour in surrounding tissues by assessment of preserved mediastinal fat planes and can detect more, and smaller, lesions [33]. In the literature, varying sensitivities and specificities can be found regarding the accurate detection of parietal pleural or chest wall invasion (T3 disease), ranging 38$90 \%$ and $40-90 \%$, respectively [34, 35]. Varying sensitivities and specificities have also been reported for the determination of whether there has been mediastinal invasion of tumour (T4 disease; $40-84 \%$ and 57-94\%, respectively) [36].
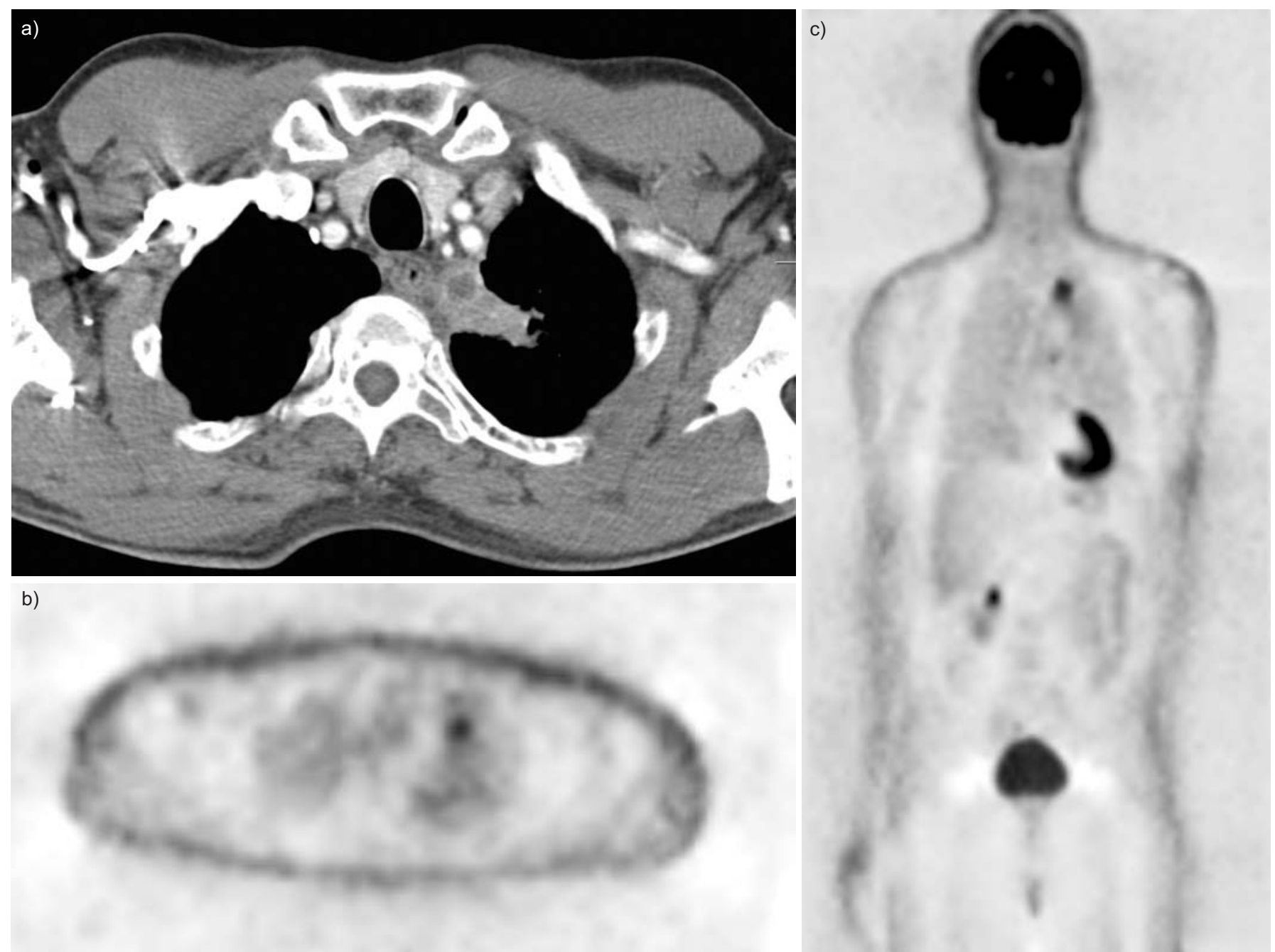

b)

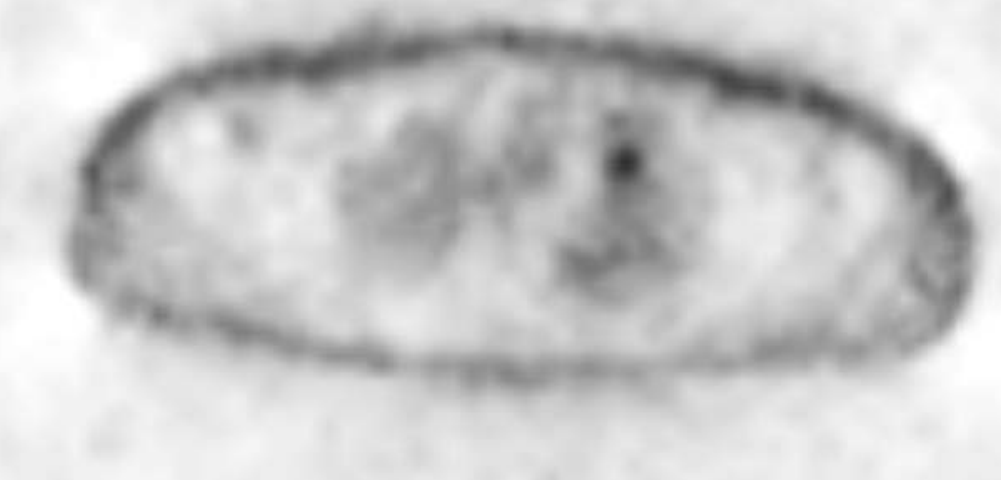

FIGURE 6. A 56-yr-old male with a lung abscess in the left upper lobe. Computed tomography (CT; a) and positron emission tomography (PET) images (b, c) show a lesion suspect for a lung tumour in the left upper lobe. However, this lesion was not a lung tumour but a lung abscess. Owing to the glucose uptake of the inflammatory processes, this lung abscess causes a false-positive finding on PET imaging. 
FDG-PET gives more information about the metabolic changes of the tumour but PET offers little extra benefit in the T-staging of lung cancer, owing its limited ability for precise anatomic location and size measurement. It is striking that PET alone both underestimates and overestimates the T-stage of many lesions. FDG not only enhances most malignant tumours but can also enhances areas of active inflammation. Conversely, some tumour tissue shows no or little FDG uptake, like microscopic tumour deposits and biologically weak tumours, such as bronchoalveolar cell carcinoma, carcinoid tumours and some adenomas [6]. In addition, PET can be helpful in evaluating the cause of pleural effusions (fig. 7) [5].

The major benefit of PET/CT lies in the direct link between the information on metabolic changes of structures and the highly detailed anatomic CT information of these structures. One of the most important attributes of PET/CT is the ability to distinguish between tumour and distal atelectasis (fig. 2). PAuls et al. [37] have found that the advantages of integrated $\mathrm{PET} / \mathrm{CT}$ also depend on the histological T-stage of the primary tumour. Changes of the therapeutic strategy due to PET/CT are especially seen in T3 and T4 tumours. In recent published studies, has been found that PET/CT is the best noninvasive imaging technique for the correct prediction of T-stage: PET/ CT correctly predicted the T-stage in $82 \%$ of cases, in comparison with 55,68 and $76 \%$ of cases when PET, CT and visual correlation of PET and CT were used, respectively. However, all imaging methods can over- and understage the tumour (table 2) [5, 6, 17, 27, 37-40].

\section{Nodal assessment ( $\mathrm{N}$-stage)}

The accuracy of CT for the prediction of intrathoracic nodal spread of tumours remains limited and the more recently developed CT systems do not change this, because nodal staging with CT is based on morphological characterisation. Lymph node size is used as the only criterion to determine metastatic disease. The current consensus considers a node $>10 \mathrm{~mm}$ in short axis diameter as suspect for metastatic lymph node [33]. Different studies with CT have shown a marked heterogeneity in the results, going from $69 \%$ to $82 \%$ for specificity and from $52 \%$ to $69 \%$ for sensitivity [41].
Over the past years, several studies have found that FDG-PET has a significantly higher sensitivity and specificity than CT in the detection of tumoural involvement of mediastinal lymph nodes. Meta-analyses have confirmed sensitivities ranging 79 $85 \%$ and specificities $89-92 \%$ [42-44]. The clinical importance of FDG-PET lies in the high negative predictive value in lymph node staging, which has been estimated as $>90 \%$ in several studies [45].

False-negative results can occur when the cancer involvement of the mediastinal nodes is low (micro metastases). Because of limitations in spatial resolution of PET, it is often not possible to distinguish between the primary tumour and the hilar nodes from adjacent mediastinal lymph nodes. Additionally, differentiation between malignant lymph node and residual brown fat or inflammatory lymph nodes can be challenging [28]. Therefore, mediastinoscopy remains the standard for mediastinal staging, even if not all mediastinal lymph nodes can be accessed with mediastinoscopy. Therefore, mediastinoscopy will still be performed at most centres if a lymph node is identified on PET as pathological [46].

Visual comparison of FDG-PET images with the CT images improves the results of lymph node staging [47]. It can be expected that diagnosis is even better when integrated PET/ CT scanners, which provide perfectly co-registered FDG-PET and CT images, are used. Initial studies demonstrated a pooled average sensitivity, specificity, positive predictive value, negative predictive value and accuracy of PET/CT of $73 \%$, $80 \%, 78 \%, 91 \%$ and $87 \%$, respectively. The results of these studies are summarised in table $3[6,17,38-40,48-50]$. The benefit of PET/CT compared with PET for nodal staging appears to lie in an increase in specificity and positive predictive value, and the benefit in accuracy of PET/CT is due to the appropriate assignment of focally increased FDG uptake. This emphasises the importance of anatomic information in conjunction with PET imaging for appropriate PET image interpretation.

\section{Assessment of distal metastases (M-stage)}

The observation of metastases in patients with NSCLC has major implications for management and prognosis. In total,
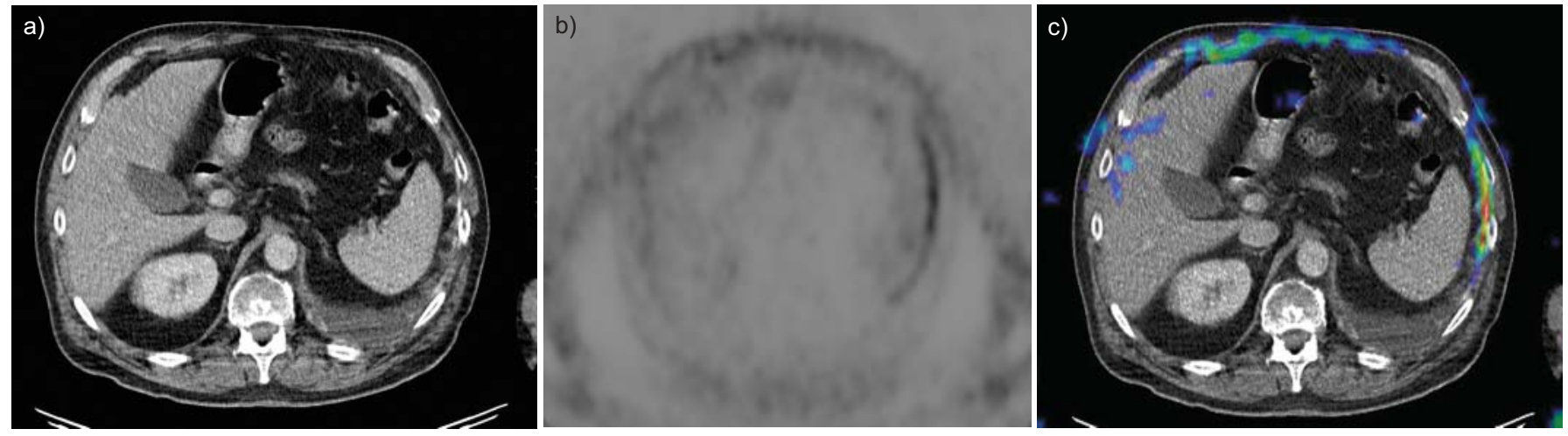

FIGURE 7. A 56-yr-old male with malignant pleuritis. a) Computed tomography (CT) shows pleural fluid in the left hemithorax with no signs of malignancy (no thickening of the pleura and no increased attenuation of the pleural fluid). b) Positron emission tomography (PET) shows an increased ${ }^{18}$ fluorodeoxyglucose (FDG) uptake in the base of the left thorax. c) Integrated PET/CT located this FDG uptake in the left costodiaphragmatic sinus on the pleura, which is suggestive for pleural metastasis; this was confirmed by thoracoscopy. 


\begin{tabular}{|c|c|c|c|c|c|c|c|c|c|c|c|}
\hline \multirow{2}{*}{$\begin{array}{l}\text { First author } \\
\text { [Ref.] }\end{array}$} & \multirow{2}{*}{$\begin{array}{c}\text { Publication } \\
\text { year }\end{array}$} & \multirow{2}{*}{ Patients n } & \multicolumn{3}{|c|}{ Correctly predicted $n$} & \multicolumn{3}{|c|}{ Overstaged $\mathbf{n}$} & \multicolumn{3}{|c|}{ Understaged $n$} \\
\hline & & & PET/CT & PET & CT & $\mathrm{PET} / \mathrm{CT}$ & PET & CT & PET/CT & PET & Ст \\
\hline ANTOCH [17] & 2003 & 16 & 15 & 12 & 12 & 1 & 3 & 2 & 0 & 1 & 2 \\
\hline Cerfolio [38] & 2004 & 91 & 64 & 43 & & 5 & 14 & & 22 & 34 & \\
\hline HaLPERN [39] & 2005 & 30 & 29 & 20 & & 0 & 1 & & 1 & 9 & \\
\hline SнIм $[40]$ & 2005 & 106 & 91 & & 84 & 4 & & 13 & 11 & & 9 \\
\hline De Wever [6] & 2007 & 50 & 42 & 23 & 34 & 4 & 8 & 10 & 3 & 19 & 6 \\
\hline Pauls [37] & 2007 & 80 & 51 & 40 & 40 & 10 & 6 & 11 & 15 & 22 & 14 \\
\hline Pfannenberg [27] & 2007 & 50 & 47 & & & 2 & & & 1 & & \\
\hline
\end{tabular}

Pooled average data are presented as n/total (\%), unless otherwise stated.

$40 \%$ of patients with NSCLC have distant metastases at presentation, most commonly in the adrenal glands, bones, liver or brain [51]. After radical treatment for seemingly localised disease, $20 \%$ of these patients develop an early distant relapse, probably due to systemic micro-metastases that were present at the time of initial staging [52].

In general, a routine search for disease beyond the chest and the upper abdomen in asymptomatic patients is not undertaken with CT and so a staging CT for lung cancer is usually done caudally from the thoracic inlet to the inferior edge of the liver, including the adrenal glands. Many reports suggest that FDG-PET is more sensitive than CT in the diagnosis of extrathoracic metastases. Sensitivity rates of $88-100 \%$ have been reported in characterising adrenal masses detected on CT in patients with lung and other primary cancers [53, 54]. Similarly, in a small series, figures of $100 \%$ sensitivity and specificity have been recorded in the detection of liver metastases from NSCLC with PET [55]. PET is also stated to be more specific than, and equally sensitive as, bone scintigraphy in the detection of bone metastases [56]. However, PET seems to be less useful in recognising brain metastases, owing to high levels of glucose uptake within normal brain tissue.The significance of isolated areas of avid FDG uptake, without anatomical reference, is uncertain and exclusion of malignancy by PET requires caution in the case of small lesions $(<1 \mathrm{~cm})$ [45].

The advantage of integrated PET/CT imaging is the ability to exactly locate a focal abnormality on PET images (fig. 3). PET/ $\mathrm{CT}$ was found to be the best noninvasive imaging technique in evaluating distant metastases in several studies [5, 6, 38, 57, 58]. CERFolio et al. [38] proved that PET/CT predicts the metastatic disease better than PET alone: $92 \%$ versus $87 \%$ correctly predicted. PET/CT and PET, respectively, correctly predicted $100 \%$ versus $86 \%$ of bone metastases, $80 \%$ versus $100 \%$ of the chest wall or pleural space metastases, $100 \%$ versus $100 \%$ of the liver metastases, $66 \%$ versus $66 \%$ of the adrenal metastases and $100 \%$ versus $50 \%$ of the gastrointestinal metastases. PET/CT identified one brain metastasis while PET missed this metastasis.

\begin{tabular}{|c|c|c|c|c|c|c|c|c|c|c|c|c|c|c|c|c|}
\hline \multirow{2}{*}{$\begin{array}{l}\text { First author } \\
\text { [Ref.] }\end{array}$} & \multirow{2}{*}{$\begin{array}{c}\text { Publication } \\
\text { year }\end{array}$} & \multicolumn{3}{|c|}{ Sensitivity \% } & \multicolumn{3}{|c|}{ Specificity \% } & \multicolumn{3}{|c|}{ PPV \% } & \multicolumn{3}{|c|}{ NPV \% } & \multicolumn{3}{|c|}{ Accuracy \% } \\
\hline & & PET/CT & PET & CT & $\mathrm{PET} / \mathrm{CT}$ & PET & CT & PET/CT & PET & CT & PET/CT & PET & CT & $\mathrm{PET} / \mathrm{CT}$ & PET & CT \\
\hline ANTOCH [17] & 2003 & 89 & 89 & 70 & 94 & 89 & 59 & 89 & 80 & 50 & 94 & 94 & 77 & 93 & 89 & 63 \\
\hline SнIм [40] & 2005 & 85 & & 70 & 84 & & 69 & & & & & & & 84 & & 69 \\
\hline Кıм [48] & 2006 & 47 & & & 100 & & & 100 & & & 87 & & & 88 & & \\
\hline De Wever [6] & 2007 & 83 & 83 & 83 & 84 & 81 & 68 & 75 & 71 & 60 & 90 & 89 & 88 & 84 & 82 & 74 \\
\hline TouRNoy [49] & 2007 & 84 & & 84 & 85 & & 61 & & & & & & & 85 & & 74 \\
\hline Y $[50]$ & 2007 & 56 & & 65 & 100 & & 89 & 100 & & 65 & 88 & & 89 & 90 & & 83 \\
\hline
\end{tabular}




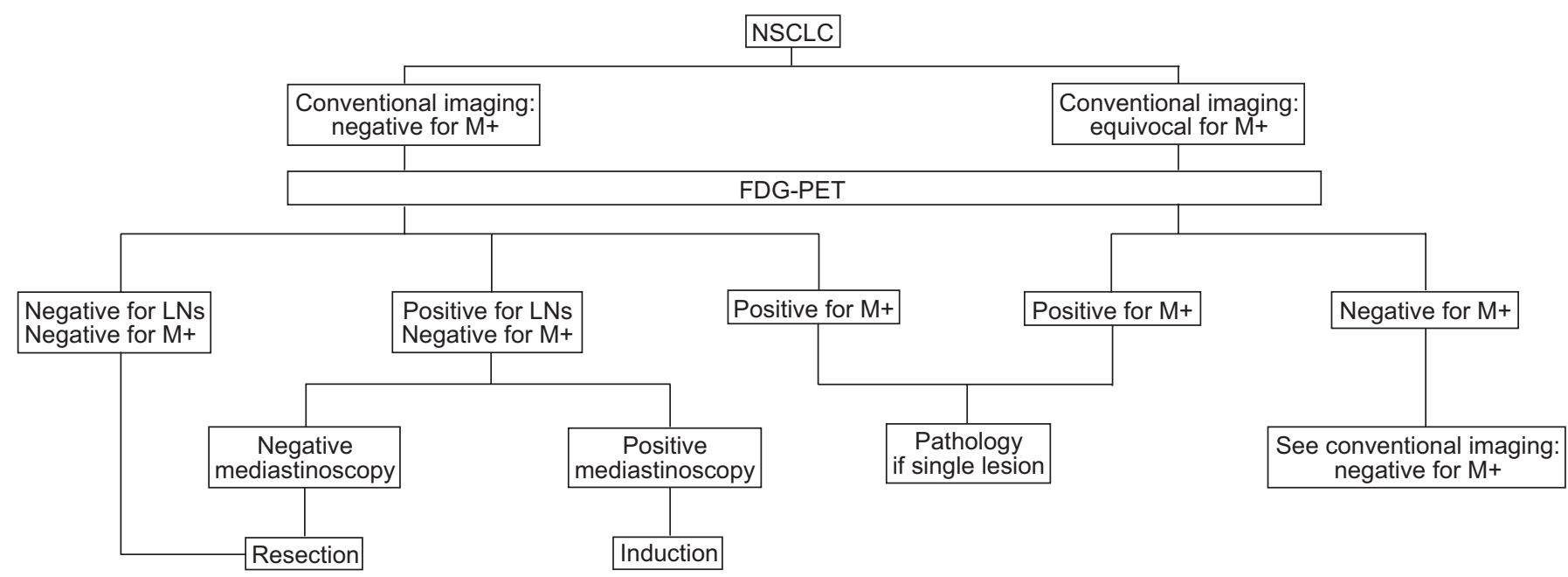

FIGURE 8. Algorithm for the staging of nonsmall cell lung cancer (NSCLC) with the implementation of positron emission tomography (PET). FDG: ${ }^{18}$ fluorodeoxyglucose; LN: Iymph nodes; M+: metastases.

\section{MEDIASTINAL RESTAGING}

Patients with stage IIIA lung cancer and in whom neoadjuvant treatment results in "downstaging" may be candidates for potentially curative surgery. While PET is very good in the initial nodal staging of lung cancer, PET alone has limited accuracy for restaging disease in the mediastinum after chemotherapy [59]. Furthermore, the results of remediastinoscopy are disappointing. DE LEYN et al. [60] have investigated this issue prospectively in 30 patients with stage IIIA N2 lung cancer. They used surgical findings as the gold standard for comparing the accuracy of PET/CT for N-staging with that of remediastinoscopy after neoadjuvant therapy. In this setting, PET/CT was more accurate than remediastinoscopy $(83 \%$ versus $60 \%$; $<<0.05$ ) and significantly more accurate than PET or CT alone. The reason for the low sensitivity of remediastinoscopy can be found in post-treatment alterations, such as adhesions and fibrosis, that rendered, especially, the subcarinal space inaccessible [60].

\section{IMPLEMENTATION OF INTEGRATED PET/CT IN THE CLINICAL PRACTICE}

Presently, both MDCT and PET are routinely used to stage patients with NSCLC in many clinical practices. A clinical algorithm, as used in University Hospitals Gasthuisberg (Leuven, Belgium) is shown in figure 8. Recently, it has become obvious that the fusion of the functional information provided by PET with the anatomic information from CT can increase the accuracy of tumour staging. The first studies using integrated PET/CT have shown that this technique improves diagnostic accuracy when compared with CT and PET alone. This improved diagnostic accuracy of integrated PET/CT is related to: 1) the detection of lesions initially not seen on CT or PET; 2) more precise location of lesions and a better delineation of the surrounding structures; and 3) a better characterisation of the lesion as benign or malignant. Another, more technical, advantage is the reduction of the PET examination time by up to $40 \%$ by using CT data for attenuation correction.

In early designs, CT and PET data acquisition and image reconstruction were performed on separate systems that accessed a common database. Increasingly, functionality has been combined to reduce costs and complexity, and to increase reliability. In the future, similar considerations of cost and complexity for the hardware may lead to greater levels of integration. There will undoubtedly be a demand for more cost-effective integrated PET/CT designs for oncology, with the likelihood that integrated PET/CT will eventually replace PET-only scanners entirely. Studies have already shown that the addition of PET to conventional workup reduces futile thoracotomies in patients with suspected potentially resectable NSCLC. The PLUS (PET in lung cancer staging) study was designed to work with routine clinical workup of patients with suspected NSCLC [61]. The conclusion of the PLUS study was also that the addition of PET to standard workup in routine clinical practice improved selection of surgically curable patients with NSCLC.

There are still issues related to how the CT portion of an integrated PET/CT examination should be performed, as well as whether i.v. and oral contrast materials are needed; however, integrated PET/CT will probably continue to gain in popularity and use in the field of oncology. It offers imaging consolidation, decreased examination time and increased throughput, as well as superior performance and exquisite location capabilities. A very important question will be: how should the CT part of the integrated PET/CT be performed, e.g. is the CT only used for attenuation correction of the PET examination or is a high-dose CT examination required in order to obtain as much anatomical detail as possible? The answer to this question is, for an important part, related to where integrated PET/CT will be placed in the clinical algorithm of lung cancer staging. As mentioned previously, a PET/CT examination can be performed using different doses for the CT. With the introduction of more advanced MDCT scanners to the integrated PET/CT machines, the diagnostic quality will further improve and may become as good as that in diagnostic CT imaging.

HERDER et al. [62] examined if the application of PET immediately after first presentation might simplify staging, 


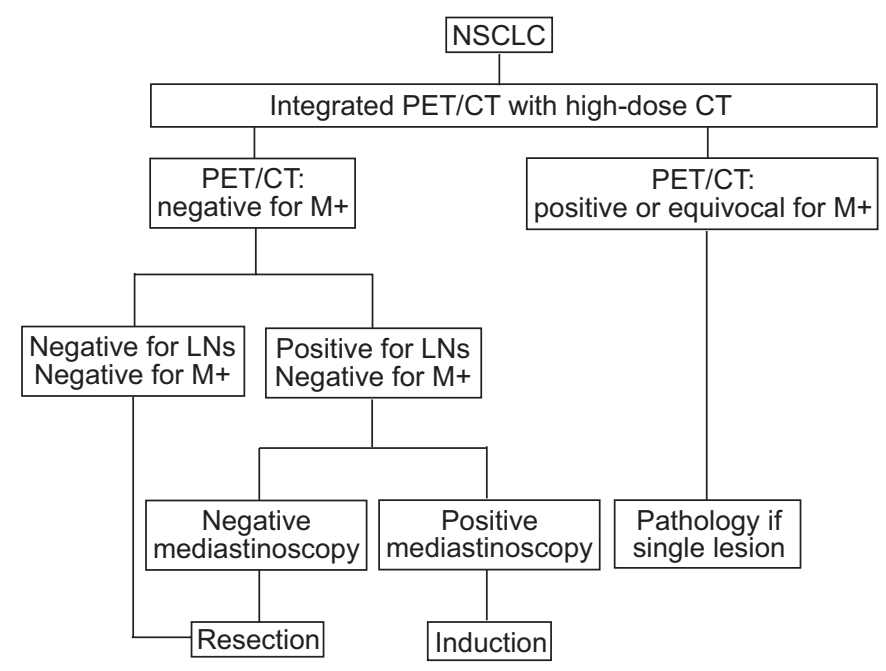

FIGURE 9. Algorithm for the staging of nonsmall cell lung cancer (NSCLC) with the implementation of integrated positron emission tomography (PET)/computed tomography (CT) with a high-dose CT. LN: lymph nodes; M+: metastases.

while maintaining accuracy, compared with the traditional strategy in routine clinical setting. The conclusion was that upfront PET in patients with (suspected) lung cancer does not reduce the overall number of diagnostic tests, but it maintains quality of TNM staging with the use of less invasive surgery. With integrated PET/CT it can be anticipated that examination costs may rise due to more expensive machinery, though this may be offset in part by improvements in staging accuracy and examination times [63]. Combining metabolic PET information with anatomic CT information in integrated PET/CT can also obviate other diagnostic tests [64]. Today, whether a high dose $\mathrm{CT}$ is performed or not depends on the moment when the patient enters the diagnostic and staging procedure. If, based on clinical evaluation, chest radiograph and bronchoscopy, suspicion of the presence of a malignant lung tumour is high, a high-dose integrated PET/CT can immediately be performed, replacing the diagnostic CT and the PET examination (fig. 9). This algorithm can be used in centres where integrated PET/ $\mathrm{CT}$ is available. Because many centres in different countries do not have integrated PET/CT, or even PET, the international guidelines, as proposed by the American Thoracic Society/ European Respiratory Society must be followed for staging patients with NSCLC [65].

\section{CONCLUSION}

Integrated PET/CT is the best imaging technique for T-staging; it is better than CT alone, PET alone and visual correlation of PET and CT. With integrated PET/CT, tumours can be better delineated from surrounding structures, such as chest wall, mediastinum or surrounding atelectasis, which is important in the exclusion of T3 or T4 stage. For N-(re)staging, integrated $\mathrm{PET} / \mathrm{CT}$ increases the specificity and positive predictive value, owing to the combination of metabolic and anatomic information. For M-staging, the additional value of integrated PET/CT is related to the fact that a CT of the whole body becomes available and to the fact that FDG hotspots can be better located. The CT part of the integrated PET/CT is often able to detect and diagnose metastatic disease, obviating specific diagnostic CT examinations while the additional FDG hotspots detected with PET are better characterised when the CT information is used. However, there are still many indeterminate lesions that need histopathological proof, and integrated $\mathrm{PET} / \mathrm{CT}$ can be helpful in guiding these interventional procedures.

With the introduction of more advanced multidetector computed tomography scanners in integrated positron emission tomography/computed tomography machines the diagnostic quality will further improve and may become as good as that in diagnostic computed tomography imaging. At that moment, integrated positron emission tomography/computed tomography will entirely replace positron emission tomography-only scanners.

\section{REFERENCES}

1 Janssen-Heijnen ML, Coebergh JW. Trends in incidence and prognosis of the histological subtypes of lung cancer in North America, Australia, New Zealand and Europe. Lung Cancer 2001; 31: 123-137.

2 Greene FL, Page DL, Fleming ID, et al. AJCC Cancer Staging Manual. 6th Edn. New York, Springer, 2002; pp. 165-177.

3 Beyer T, Townsend DW, Brun T, et al. A combined PET/CT scanner for clinical oncology. J Nucl Med 2000; 41: 1369-1379.

4 Bar-Shalom R, Yefremov N, Guralnik L, et al. Clinical performance of PET/CT in evaluation of cancer: additional value for diagnostic imaging and patient management. $J$ Nucl Med 2003; 44: 1200-1209.

5 Lardinois D, Weder W, Hany TF, et al. Staging of nonsmall-cell lung cancer with integrated positron-emission tomography and computed tomography. $N$ Engl J Med 2003; 348: 2500-2507.

6 De Wever W, Ceyssens S, Mortelmans L, et al. Additional value of PET-CT in the staging of lung cancer: comparison with CT alone, PET alone and visual correlation of PET and CT. Eur Radiol 2007; 17: 23-32.

7 Dahlbom M, Hoffman EJ, Hoh CK, et al. Whole-body positron emission tomography: Part I. Methods and performance characteristics. J Nucl Med 1992; 33: 1191-1199.

8 Burger C, Goerres G, Schoenes S, Buck A, Lonn AH, Von Schulthess GK. PET attenuation coefficients from CT images: experimental evaluation of the transformation of CT into PET 511-keV attenuation coefficients. Eur J Nucl Med Mol Imaging 2002; 29: 922-927.

9 Yau YY, Chan WS, Tam YM, et al. Application of intravenous contrast in PET/CT: does it really introduce significant attenuation correction error? J Nucl Med 2005; 46: $283-291$.

10 Gilman MD, Fischman AJ, Krishnasetty V, Halpern EF, Aquino SL. Optimal CT breathing protocol for combined thoracic PET/CT. AJR Am J Roentgenol 2006; 187: 1357-1360.

11 Osman MM, Cohade C, Nakamoto Y, Wahl RL. Respiratory motion artifacts on PET emission images obtained using CT attenuation correction on PET-CT. Eur J Nucl Med Mol Imaging 2003; 30: 603-606.

12 Rosenbaum SJ, Lind T, Antoch G, Bockisch A. Falsepositive FDG PET uptake - the role of PET/CT. Eur Radiol 2006; 16: 1054-1065. 
13 Goerres GW, Kamel E, Heidelberg TN, Schwitter MR, Burger C, von Schulthess GK. PET-CT image co-registration in the thorax: influence of respiration. Eur J Nucl Med Mol Imaging 2002; 29: 351-360.

14 de Juan R, Seifert B, Berthold T, von Schulthess GK, Goerres GW. Clinical evaluation of a breathing protocol for PET/CT. Eur Radiol 2004; 14: 1118-1123.

15 Beyer T, Antoch G, Blodgett T, Freudenberg LF, Akhurst T, Mueller S. Dual-modality PET/CT imaging: the effect of respiratory motion on combined image quality in clinical oncology. Eur J Nucl Med Mol Imaging 2003; 30: 588-596.

16 Bockisch A, Beyer T, Antoch G, et al. Positron emission tomography/computed tomography - imaging protocols, artifacts, and pitfalls. Mol Imaging Biol 2004; 6: 188-199.

17 Antoch G, Stattaus J, Nemat AT, et al. Non-small cell lung cancer: dual-modality PET/CT in preoperative staging. Radiology 2003; 229: 526-533.

18 Antoch G, Freudenberg LS, Beyer T, Bockisch A, Debatin JF. To enhance or not to enhance? 18F-FDG and CT contrast agents in dual-modality 18F-FDG PET/CT. J Nucl Med 2004; 45: Suppl. 1, 56S-65S.

19 Antoch G, Freudenberg LS, Stattaus J, et al. Whole-body positron emission tomography-CT: optimized CT using oral and IV contrast materials. AJR Am J Roentgenol 2002; 179: 1555-1560.

20 Cohade C, Osman M, Nakamoto Y, et al. Initial experience with oral contrast in PET/CT: phantom and clinical studies. J Nucl Med 2003; 44: 412-416.

21 Dizendorf E, Hany TF, Buck A, von Schulthess GK, Burger C. Cause and magnitude of the error induced by oral CT contrast agent in CT-based attenuation correction of PET emission studies. J Nucl Med 2003; 44: 732-738.

22 Hany TF, Steinert HC, Goerres GW, Buck A, von Schulthess GK. PET diagnostic accuracy: improvement with in-line PET-CT system: initial results. Radiology 2002; 225: 575-581.

23 Brix G, Lechel U, Glatting G, et al. Radiation exposure of patients undergoing whole-body dual-modality 18F-FDG PET/CT examinations. J Nucl Med 2005; 46: 608-613.

24 Kalra MK, Maher MM, Toth TL, et al. Strategies for CT radiation dose optimization. Radiology 2004; 230: 619-628.

25 Brix G, Nagel HD, Stamm G, et al. Radiation exposure in multi-slice versus single-slice spiral CT: results of a nationwide survey. Eur Radiol 2003; 13: 1979-1991.

26 Kuehl H, Veit P, Rosenbaum SJ, Bockisch A, Antoch G. Can $\mathrm{PET} / \mathrm{CT}$ replace separate diagnostic CT for cancer imaging? Optimizing CT protocols for imaging cancers of the chest and abdomen. J Nucl Med 2007; 48: Suppl. 1, 45S-57S.

27 Pfannenberg AC, Aschoff P, Brechtel K, et al. Low dose non-enhanced CT versus standard dose contrast-enhanced CT in combined PET/CT protocols for staging and therapy planning in non-small cell lung cancer. Eur J Nucl Med Mol Imaging 2007; 34: 36-44.

28 Truong MT, Erasmus JJ, Macapinlac HA, et al. Integrated positron emission tomography/computed tomography in patients with non-small cell lung cancer: normal variants and pitfalls. J Comput Assist Tomogr 2005; 29: 205-209.

29 Kubota R, Yamada S, Kubota K, Ishiwata K, Tamahashi N, Ido T. Intratumoral distribution of fluorine-18-fluorodeoxyglucose in vivo: high accumulation in macrophages and granulation tissues studied by microautoradiography. J Nucl Med 1992; 33: 1972-1980.

30 Ogawa M, Ishino S, Mukai T, et al. ${ }^{18}$ F-FDG accumulation in atherosclerotic plaques: immunohistochemical and PET imaging study. J Nucl Med 2004; 45: 1245-1250.

31 Hany TF, Heuberger J, von Schulthess GK. Iatrogenic FDG foci in the lungs: a pitfall of PET image interpretation. Eur Radiol 2003; 13: 2122-2127.

32 Shim SS, Lee KS, Kim BT, Choi JY, Chung MJ, Lee EJ. Focal parenchymal lung lesions showing a potential of falsepositive and false-negative interpretations on integrated PET/CT. AJR Am J Roentgenol 2006; 186: 639-648.

33 Verschakelen JA, De Wever W, Bogaert J. Role of computed tomography in lung cancer staging. Curr Opin Pulm Med 2004; 10: 248-255.

34 Quint LE, Francis IR. Radiologic staging of lung cancer. J Thorac Imaging 1999; 14: 235-246.

35 Mori K, Hirose T, Machida S, et al. Helical computed tomography diagnosis of pleural dissemination in lung cancer: comparison of thick-section and thin-section helical computed tomography. J Thorac Imaging 1998; 13: 211-218.

36 Munden RF, Swisher SS, Stevens CW, Stewart DJ. Imaging of the patient with non-small cell lung cancer. Radiology 2005; 237: 803-818.

37 Pauls S, Buck AK, Hohl K, et al. Improved non-invasive Tstaging in non-small cell lung cancer by integrated $18 \mathrm{~F}$ FDG PET/CT. Nuklearmedizin 2007; 46: 9-14.

38 Cerfolio RJ, Ojha B, Bryant AS, Raghuveer V, Mountz JM, Bartolucci AA. The accuracy of integrated PET-CT compared with dedicated PET alone for the staging of patients with nonsmall cell lung cancer. Ann Thorac Surg 2004; 78: 1017-1023.

39 Halpern BS, Schiepers C, Weber WA, et al. Presurgical staging of non-small cell lung cancer: positron emission tomography, integrated positron emission tomography/ CT, and software image fusion. Chest 2005; 128: 2289-2297.

40 Shim SS, Lee KS, Kim BT, et al. Non-small cell lung cancer: prospective comparison of integrated FDG PET/CT and CT alone for preoperative staging. Radiology 2005; 236: 1011-1019.

41 Dillemans B, Deneffe G, Verschakelen J, Decramer M. Value of computed tomography and mediastinoscopy in preoperative evaluation of mediastinal nodes in non-small cell lung cancer. A study of 569 patients. Eur J Cardiothorac Surg 1994; 8: 37-42.

42 Birim O, Kappetein AP, Stijnen T, Bogers AJ. Meta-analysis of positron emission tomographic and computed tomographic imaging in detecting mediastinal lymph node metastases in nonsmall cell lung cancer. Ann Thorac Surg 2005; 79: 375-382.

43 Dwamena BA, Sonnad SS, Angobaldo JO, Wahl RL. Metastases from non-small cell lung cancer: mediastinal staging in the 1990s - meta-analytic comparison of PET and CT. Radiology 1999; 213: 530-536.

44 Gould MK, Kuschner WG, Rydzak CE, et al. Test performance of positron emission tomography and computed tomography for mediastinal staging in patients with non-small cell lung cancer: a meta-analysis. Ann Intern Med 2003; 139: 879-892. 
45 Schrevens L, Lorent N, Dooms C, Vansteenkiste J. The role of PET scan in diagnosis, staging, and management of nonsmall cell lung cancer. Oncologist 2004; 9: 633-643.

46 Yasufuku K, Fujisawa T. Staging and diagnosis of nonsmall cell lung cancer: invasive modalities. Respirology 2007; 12: 173-183.

47 Aquino SL, Asmuth JC, Alpert NM, Halpern EF, Fischman AJ. Improved radiologic staging of lung cancer with 2-[18F]-fluoro-2-deoxy-D-glucose-positron emission tomography and computed tomography registration. J Comput Assist Tomogr 2003; 27: 479-484.

48 Kim BT, Lee KS, Shim SS, et al. Stage T1 non-small cell lung cancer: preoperative mediastinal nodal staging with integrated FDG PET/CT - a prospective study. Radiology 2006; 241: 501-509.

49 Tournoy KG, Maddens S, Gosselin R, Van Maele G, van Meerbeeck JP, Kelles A. Integrated FDG-PET/CT does not make invasive staging of the intrathoracic lymph nodes in non-small cell lung cancer redundant: a prospective study. Thorax 2007; 62: 696-701.

50 Yi CA, Lee KS, Kim BT, et al. Efficacy of helical dynamic CT versus integrated PET/CT for detection of mediastinal nodal metastasis in non-small cell lung cancer. AJR Am J Roentgenol 2007; 188: 318-325.

51 Quint LE, Tummala S, Brisson LJ, et al. Distribution of distant metastases from newly diagnosed non-small cell lung cancer. Ann Thorac Surg 1996; 62: 246-250.

52 Pantel K, Izbicki J, Passlick B, et al. Frequency and prognostic significance of isolated tumour cells in bone marrow of patients with non-small cell lung cancer without overt metastases. Lancet 1996; 347: 649-653.

53 Jana S, Zhang T, Milstein DM, Isasi CR, Blaufox MD. FDGPET and CT characterization of adrenal lesions in cancer patients. Eur J Nucl Med Mol Imaging 2006; 33: 29-35.

54 Kumar R, Xiu Y, Yu JQ, et al. 18F-FDG PET in evaluation of adrenal lesions in patients with lung cancer. J Nucl Med 2004; 45: 2058-2062.

55 Marom EM, McAdams HP, Erasmus JJ, et al. Staging nonsmall cell lung cancer with whole-body PET. Radiology 1999; 212: 803-809.

56 Bury T, Barreto A, Daenen F, Barthelemy N, Ghaye B, Rigo P. Fluorine-18 deoxyglucose positron emission tomography for the detection of bone metastases in patients with non-small cell lung cancer. Eur J Nucl Med 1998; 25: 1244-1247.

57 De Wever W, Vankan Y, Stroobants S, Verschakelen J. Detection of extrapulmonary lesions with integrated PET/CT in the staging of lung cancer. Eur Respir J 2007; 29: 995-1002.

58 Lardinois D, Weder W, Roudas M, et al. Etiology of solitary extrapulmonary positron emission tomography and computed tomography findings in patients with lung cancer. $J$ Clin Oncol 2005; 23: 6846-6853.

59 Hoekstra CJ, Stroobants SG, Smit EF, et al. Prognostic relevance of response evaluation using [18F]-2-fluoro-2deoxy-D-glucose positron emission tomography in patients with locally advanced non-small cell lung cancer. J Clin Oncol 2005; 23: 8362-8370.

60 De Leyn P, Stroobants S, De Wever W, et al. Prospective comparative study of integrated positron emission tomography-computed tomography scan compared with remediastinoscopy in the assessment of residual mediastinal lymph node disease after induction chemotherapy for mediastinoscopy-proven stage IIIA-N2 non-small cell lung cancer: a Leuven Lung Cancer Group Study. J Clin Oncol 2006; 24: 3333-3339.

61 van Tinteren H, Hoekstra OS, Smit EF, et al. Effectiveness of positron emission tomography in the preoperative assessment of patients with suspected non-small cell lung cancer: the PLUS multicentre randomised trial. Lancet 2002; 359: 1388-1393.

62 Herder GJ, Kramer H, Hoekstra OS, et al. Traditional versus up-front $[18 \mathrm{~F}]$ fluorodeoxyglucose-positron emission tomography staging of non-small cell lung cancer: a Dutch cooperative randomized study. J Clin Oncol 2006; 24: $1800-1806$.

63 Devaraj A, Cook GJ, Hansell DM. PET/CT in non-small cell lung cancer staging - promises and problems. Clin Radiol 2007; 62: 97-108.

64 De Wever W. Role of integrated PET/CT in the staging of non-small lung cancer. PhD thesis. KU Leuven, Leuven, Belgium, 2008.

65 Pretreatment evaluation of non-small-cell lung cancer. The American Thoracic Society and The European Respiratory Society. Am J Respir Crit Care Med 1997; 156: 320-332. 